\title{
Measuring Transient Reaction Rates from Nonstationary Catalysts
}

\author{
Dmitriy Borodin, Kai Golibrzuch, Michael Schwarzer, Jan Fingerhut, Georgios Skoulatakis, \\ Dirk Schwarzer, Thomas Seelemann, Theofanis Kitsopoulos,* and Alec M. Wodtke*
}

Cite This: ACS Catal. 2020, 10, 14056-14066

Read Online

ACCESS | LWl Metrics \& More | 回 Article Recommendations

ABSTRACT: Up to now, methods for measuring rates of reactions on catalysts required long measurement times involving signal averaging over many experiments. This imposed a requirement that the catalyst return to its original state at the end of each experimenta complete reversibility requirement. For real catalysts, fulfilling the reversibility requirement is often impossible-catalysts under reaction conditions may change their chemical composition and structure as they become activated or while they are being poisoned through use. It is therefore desirable to develop high-speed methods where transient rates can be quickly measured while catalysts are changing. In this work, we present velocity-resolved kinetics using high-repetition-rate pulsed laser ionization and high-speed ion imaging detection. The reaction is initiated by a single molecular beam pulse incident at the surface, and the product formation rate is observed by a sequence of pulses produced by a high-repetition-rate laser. Ion imaging provides the desorbing product flux (reaction rate) as a function of reaction time for each laser pulse. We demonstrate the principle of this approach by rate measurements on two simple reactions: $\mathrm{CO}$ desorption from and $\mathrm{CO}$ oxidation on the 332 facet of $\mathrm{Pd}$. This approach overcomes the time-consuming scanning of the delay between $\mathrm{CO}$ and laser pulses needed in past experiments and delivers a data acquisition rate that is 10-1000 times higher. We are able to record kinetic traces of $\mathrm{CO}_{2}$ formation while a $\mathrm{CO}$ beam titrates oxygen atoms from an O-saturated surface. This approach also allows measurements of reaction rates under diffusion-controlled conditions.

KEYWORDS: high-speed imaging, velocity-resolved kinetics, CO oxidation, molecular beams, heterogeneous catalysis

\section{INTRODUCTION}

Methods to measure the kinetics of surface reactions are fundamental to improving our understanding of heterogeneous catalysis. Traditionally, temperature-programmed reaction, molecular beam relaxation spectrometry, and phase-lag detection have been available to experimentalists. ${ }^{1-4}$ Recently, the kinetic trace was obtained using velocity-resolved methods ${ }^{5}$ based on ion imaging. ${ }^{6-8}$ This is essentially a pump-probe technique where a molecular beam pump-pulse initiates the reaction and pulsed laser ionization probes the desorbing products. Varying the delay between the two pulses provides the time base of the reaction kinetics. The ionized products are recorded with ion imaging providing product velocity information with every detection pulse. This allows measured product densities to be converted to product flux, which is by definition the reaction rate for a surface reaction. Furthermore, flight times irrelevant to the reaction time can be subtracted from the experimental time axis. ${ }^{9}$ Like all pump-probe measurements, during the time that the delay between pump and probe is being scanned, the catalyst under study must not change-furthermore, it must return to its original state between each pump-probe experiment. However, catalysts are often dynamic. Catalyst composition can change dramatically under reactive conditions ${ }^{10}$ - living catalyst ${ }^{11-13}$ - and catalytic use can lead to poisoning. ${ }^{14}$ Hence, we need methods that can rapidly obtain kinetic information, providing real-time rates on a measurement time scale that is faster than the speed with which the catalyst is changing.

In this work, we demonstrate velocity-resolved kinetics with high-repetition-rate detection. The reaction starts when a pulse of molecules arrives at the surface, and ion images are recorded for each pulse of a high-repetition-rate laser that ionizes desorbing products. The ion images preserve the velocity information from which the rate of reaction is derived. The inverse repetition rate of the laser sets the temporal resolution. We demonstrate a duty cycle that is $1-3$ orders of magnitude higher than previous methods, ${ }^{5}$ allowing measurements on a changing catalyst. The present experiments use a $1 \mathrm{kHz}$ Ti:sapphire laser-future experiments with Yb-fiber lasers operating at $10^{2-3} \mathrm{kHz}$ provide a perspective for improvement.

Received: August 29, 2020

Revised: November 6, 2020

Published: November 17, 2020
Catalysis

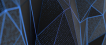


(a) Velocity Resolved Kinetics - Delay Scanning

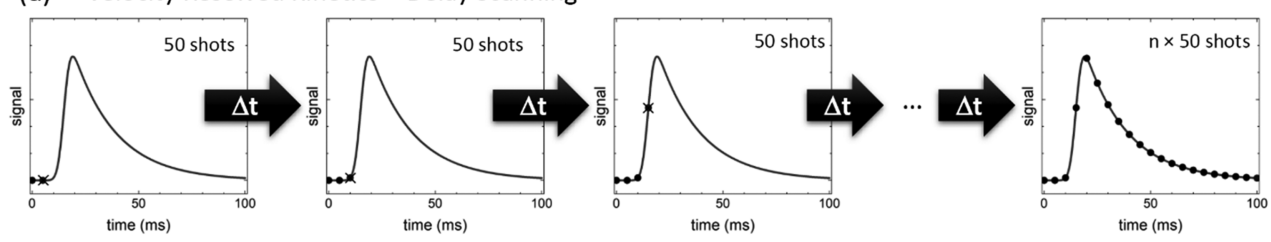

(b) Velocity Resolved Kinetics - High rep-rate detection

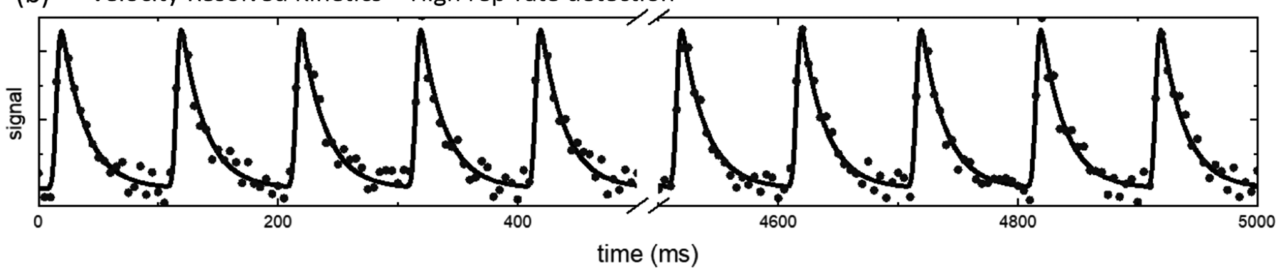

Figure 1. Comparison of delay scanning versus high-rep-rate detection employed in velocity-resolved kinetics measurements. (a) Delay scanning involves the acquisition of many (e.g., 50) images at each time delay between the initiating molecular beam pulse and the laser ionization pulse. Points in the kinetic trace recorded by scanning the delay between a molecular beam pulse that initiates the reaction and a laser ionization pulse that detects the products. The catalytic system must be stable throughout the course of the delay scanning procedure. (b) High-rep-rate detection with high-speed imaging records many points in the kinetic trace for each molecular beam pulse. Here, the molecular beam initiates the reaction every $0.1 \mathrm{~s}$ and points in the kinetic trace are recorded by each pulse of a $1 \mathrm{kHz}$ detection laser. The duty cycle of this method can be much higher than delay scanning. Furthermore, the kinetics can be recorded while the catalyst is changing.

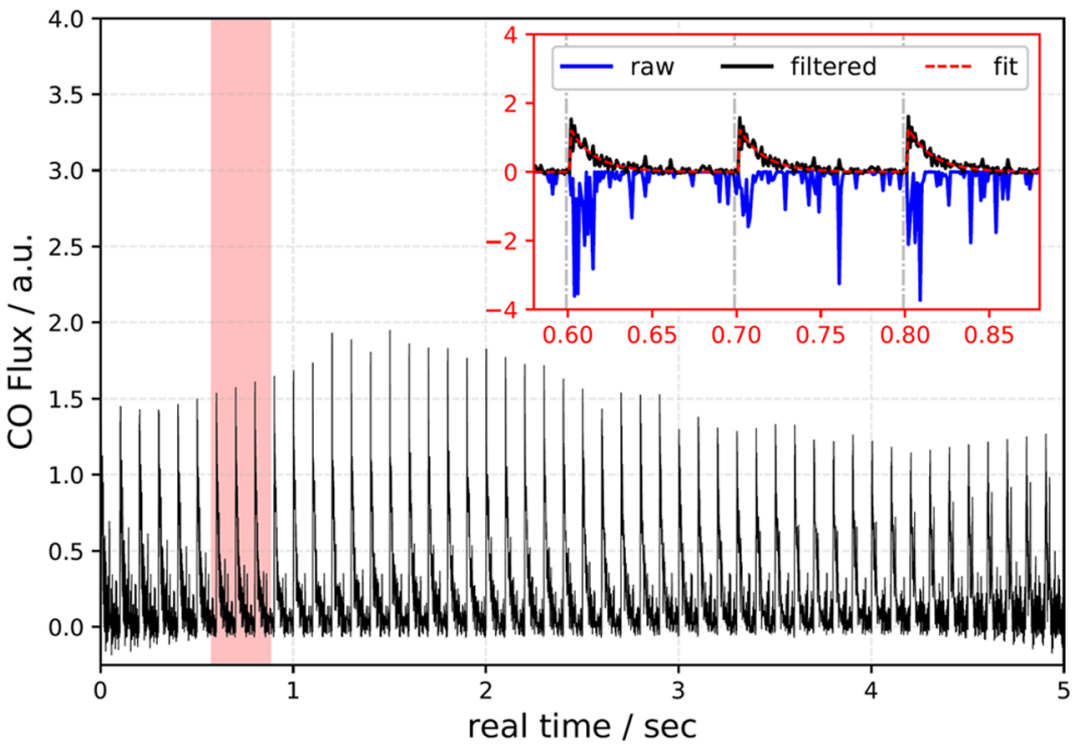

Figure 2. CO trapping/desorption from $\mathrm{Pd}(332)$ as a proof of principle for high-rep-rate detection. The CO pulsed beam runs at $10 \mathrm{~Hz}$, while the detection laser runs at $1 \mathrm{kHz}$. The Pd crystal was held at $593 \mathrm{~K}$. Inset: Raw data (blue line) is treated by the Savitzky-Golay filter (see text) to yield the filtered data (black line). The gray dash-dotted line indicates the time at which the CO pulse initiates the reaction. The dashed red line is a periodic first-order decay convoluted with the temporal profile of the $\mathrm{CO}$ beam and is used to extract the desorption rate constant.

\section{EXPERIMENT}

We previously described the apparatus in detail elsewhere. ${ }^{6-8}$ Briefly, we produce two molecular beams in two vacuum chambers, each equipped with piezo-electrically actuated pulsed valves. The valves' repetition rates are variable up to $500 \mathrm{~Hz}$. The pulse durations can be as low as $30 \mu \mathrm{s}$. Each beam passes through two differential pumping chambers, before entering an ultrahigh vacuum (UHV) chamber with a base pressure of $2 \times 10^{-10} \mathrm{mbar}$, where they intersect with one another and collide with a $\operatorname{Pd}(332)$ surface. One beam collides at normal incidence dosing the sample with oxygen. The second beam, incident at $30^{\circ}$ to the normal, initiates the reaction with a pulse of $\mathrm{CO}$. The $\mathrm{CO}$ beam can either be used alone to study $\mathrm{CO}$ trapping/desorption or with an oxidized surface to initiate $\mathrm{CO}_{2}$ formation. A single crystal of Pd cut and polished to expose the (332) surface is mounted on a 5-axis manipulator and can be heated to $1150 \mathrm{~K}$ using electron bombardment. The instrument is equipped with an $\mathrm{Ar}^{+}$ sputtering source for cleaning the surface as well as an Auger electron spectrometer to check its cleanliness.

A homogeneous electric field oriented parallel to the surface is formed by two parallel flat meshes (repeller and extractor), between which both molecular beams pass. After ionization of the reaction products by a nonresonant multiphoton process, using an ultrashort Ti:sapphire laser (Coherent Astrella, 800 $\mathrm{nm}, 35 \mathrm{fs}, 0.5 \mathrm{~mJ}, 1 \mathrm{kHz}$ ) focused with a $150 \mathrm{~mm}$ plano-convex lens, a $3 \mathrm{kV}$ pulse applied to the repeller of the ion imaging system directs the ions to the imaging detector. This maps the products' density and in-plane velocity vectors, which is used 
to create a flux image. A region of the flux image is then integrated to provide the rate of reaction at a specific time. We record ion images with a $56 \mathrm{~mm}$ Chevron MCP detector coupled to a P43 phosphor screen, whose phosphorescence detected by a high-frame-rate CMOS camera (Vision Research Phantom VEO 710). We took advantage of commercial data acquisition software (DaVis LaVision $\mathrm{GmbH}$ ) and a softwarecontrolled timing unit (PTUX, LaVision $\mathrm{GmbH}$ ). The timing unit is triggered both at $10 \mathrm{~Hz}$-synchronized with the pulsed nozzle-and at $1 \mathrm{kHz}$ - synchronized with the laser. Several thousand images are recorded over several seconds and stored on the camera's internal memory, only to be transferred later to a computer's hard disk.

Figure 1 shows a comparison to methods requiring the delay between pulsed molecular beam and laser, $t_{\mathrm{BL}}$, to be scanned. In that case (Figure 1a), an ion image is measured for a fixed value $t_{\mathrm{BL}}$ and an ion image is accumulated over many (typically 50) molecular beam pulses. $t_{\mathrm{BL}}$ is then incremented and the process is repeated. Here, one ion image is recorded for every molecular beam pulse, whose repetition rate is typically 10$100 \mathrm{~Hz}$. Using high-repetition-rate detection, the ion image is recorded every millisecond. Each pulse of the laser (points in Figure $1 \mathrm{~b}$ ) corresponds to a point in the temporal evolution of the reaction. The P43 phosphor screen decays over $\tau_{90 \rightarrow 10 \%}=$ $1.3 \mathrm{~ms}$, while the time between laser pulses is only $1 \mathrm{~ms}$. Hence, after downloading the image sequence to the computer, we subtracted from each image the "afterglow background" remaining from the previous image.

\section{RESULTS AND DISCUSSION}

\subsection{Proof of Principle: Application to CO Desorption} from $\mathrm{Pd}(332)$. As a proof of principle, we performed measurements on CO trapping/desorption from $\mathrm{Pd}(332)$ between 583 and $623 \mathrm{~K}$. Here, a clean $\operatorname{Pd}(332)$ crystal is exposed to a pulsed molecular beam of pure $\mathrm{CO}$ operating at $10 \mathrm{~Hz}$. The surface temperature is controlled so that a $1 \mathrm{kHz}$ detection rate is sufficiently rapid to follow the desorption kinetics, while also ensuring that all $\mathrm{CO}$ molecules desorb between molecular beam pulses. Following refs 6, 7, we extract the kinetic trace by integrating flux images between 300 and $900 \mathrm{~m} / \mathrm{s}$ and $\pm 4^{\circ}$ from the surface normal. This captures most of the desorbing molecules, while suppressing signal from directly scattered (higher velocity) and background (lower velocity) $\mathrm{CO}$.

Figure 2 shows data from a typical $5 \mathrm{~s}$ experiment, requiring $1 \%$ the measurement time needed for delay scanning. Fifty kinetic traces result, one from each of $50 \mathrm{CO}$ molecular beam pulses. The inset shows three kinetic traces in detail. We filter the raw data (blue) with a periodic Savitzky-Golay filter ${ }^{15}$ applied by first sorting the data according to $t_{\mathrm{BL}}$ (the delay between $\mathrm{CO}$ beam pulse and ionizing laser pulse) and then employing a moving linear fit to a single data point and 10 of its neighboring data points-all with the same $t_{\mathrm{BL}}$. The value of the fitted line then replaces the data point, and the process is repeated on the next data point. This leads to the filtered output (black). The CO desorption rate constant, $k_{\mathrm{d}}$ is determined by fitting each pulsed decay with a function that convolves the incident $\mathrm{CO}$ beam's temporal profile with an exponential decay-red dashed line in the inset of Figure $2 .{ }^{5}$ In this way, we derive 50 independent values of $k_{d}$, from which we obtain an average value and a standard deviation.

Figure 3 shows $k_{\mathrm{d}}$ values for $\mathrm{CO}$ on $\mathrm{Pd}(332)$ and $\operatorname{Pd}(111)$ using several different methods. The rate constants obtained

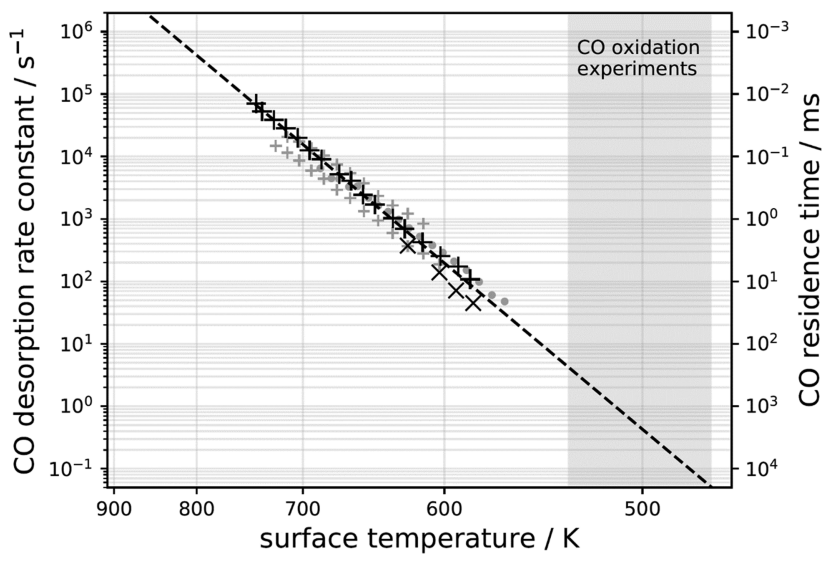

Figure 3. Desorption rate constants of $\mathrm{CO}$ from $\mathrm{Pd}(332)$ and $\operatorname{Pd}(111)$ vs surface temperature. $X$ indicates values obtained from high-rep-rate detection; + indicates results from delay scanning gray plus used delay scanning with $\operatorname{Pd}(111){ }^{7}$ and gray circle solid indicates results on $\mathrm{Pd}(111)$ from modulated molecular beam spectrometry. ${ }^{16}$ The black dashed line is an Arrhenius fit $\left(A=10^{15.6 \pm 0.3} \mathrm{~s}^{-1}\right.$ and $E_{\mathrm{a}}=$ $1.58 \pm 0.02 \mathrm{eV})$ to all desorption rate constants on $\mathrm{Pd}(332)$. The gray shaded region indicates the temperature range at which $\mathrm{CO}$ oxidation measurements in this work are conducted-see Section 3.3. Uncertainties in the rate constants determined from delay scanning and high-rep-rate detection are smaller than the symbols.

from the data of Figure $2(x)$ are in good agreement with other methods. We note that the observed desorption rates depend little on the presence of atomic steps that are found in high concentration on the $\operatorname{Pd}(332)$ surface. ${ }^{7,16}$ Clearly, steps do not significantly stabilize $\mathrm{CO}$ on $\mathrm{Pd}$, a conclusion that is consistent with reported isosteric heats of adsorption. ${ }^{17}$ An Arrhenius fit to $k_{\mathrm{d}}$ values using $\operatorname{Pd}(332)$ results yields $E_{\mathrm{a}}=1.58$ $\pm 0.02 \mathrm{eV}$ and $A=10^{15.6 \pm 0.3} \mathrm{~s}^{-1}$.

3.2. Duty Cycle Analysis. We consider now the quantitative duty cycle improvements possible with high-reprate detection, within the specific context of desorption rates near zero coverage. We first define a characteristic desorption time, $\tau$, which is the inverse of the desorption rate constant, $\tau=$ $k_{\mathrm{d}}^{-1}$. This imposes an upper limit of the molecular beam's repetition rate $\left(f_{\mathrm{MB}}^{\max }\right)$ and therefore a minimum repeat time, $t_{\min }=1 / f_{\mathrm{MB}}^{\max }$, needed to maintain the low-coverage condition. While there is some ambiguity involved, we set $t_{\min }=5 \tau$, the time at which a first-order decay has reached $0.7 \%$ of its initial value. Data obtained within $t_{\min }$ are most important to the fitting-we label this data "relevant".

The number of relevant data obtained from each molecular beam pulse used in the high-rep-rate approach, $n_{\mathrm{HRR}}$ is given by

$$
n_{\mathrm{HRR}}=t_{\min } \times f_{\mathrm{L}}
$$

where $f_{\mathrm{L}}$ is the detection laser repetition rate and data acquisition rate, $\dot{n}_{\mathrm{HRR}}$ is given by

$$
\dot{n}_{\mathrm{HRR}}=t_{\text {min }} \times f_{\mathrm{L}} \times f_{\mathrm{MB}}
$$

where $f_{\mathrm{MB}}$ is the repetition rate of the pulsed molecular beam.

The number of relevant data per molecular beam pulse in a conventional delay scanning experiments, $n_{\mathrm{DS}}$, is 1 , and the data acquisition rate is then

$$
\dot{n}_{\mathrm{DS}}=f_{\mathrm{MB}}
$$


Taking the ratio of these two data acquisition rates, we find that the theoretical improvement in duty cycle is given by

$$
\frac{\dot{n}_{\mathrm{HRR}}^{\max }}{\dot{n}_{\mathrm{DS}}^{\max }}=t_{\min } \times f_{\mathrm{L}}=f_{\mathrm{L}} / f_{\mathrm{MB}}^{\max }
$$

The data acquisition rate using the delay scanning approach is limited by $t_{\mathrm{min}}^{-1}$ and, of course, technical limitations to the reprate of pulse beams (in our experience, $\sim 500 \mathrm{~Hz}$ ), whereas $f_{\mathrm{L}}$ is the only limiting factor to the data acquisition rate for the high-rep-rate method. We emphasize that $f_{\mathrm{L}}$ can be improved dramatically. This work used a Ti:sapphire laser, $f_{\mathrm{L}}=1 \mathrm{kHz}$; newly available $\mathrm{Yb}$-fiber lasers achieve repetition rates of $10^{2}-$ $10^{3} \mathrm{kHz}$, while still providing pulse energies and peak intensities sufficient for nonresonant multiphoton ionization.

The analysis so far neglects the number of ions produced in each experiment, which is equally important as the rate of data acquisition. All velocity-resolved kinetics signals are proportional to the rate of product formation. Hence, in the desorption experiments presented above, the number of ions detected per laser pulse is proportional to $1 / \tau$. The dependence on $\tau$ reflects the temporal dilution seen for slow reactions. Each molecular beam pulse deposits the same number of $\mathrm{CO}$ molecules on the surface; so, the observed density is diluted greatly over time for slow reactions and less so for fast reactions. Taking this into account, we may define the "count acquisition rate" (CAR).

$$
\begin{aligned}
& \mathrm{CAR} \equiv \frac{\dot{n}}{\tau}=\dot{n} \times k_{\mathrm{d}} \\
& \mathrm{CAR}_{\mathrm{DS}} \equiv \frac{f_{\mathrm{MB}}}{\tau} \\
& \mathrm{CAR}_{\mathrm{HRR}} \equiv \frac{5 \tau \times f_{\mathrm{L}} \times f_{\mathrm{MB}}}{\tau}=5 \times f_{\mathrm{MB}} \times f_{\mathrm{L}}
\end{aligned}
$$

This quantity determines the signal-to-noise ratio $(\mathrm{S} / \mathrm{N})$ of the data obtained in any experiment. These equations point out that experiments using delay scans exhibit a stronger decrease of $\mathrm{S} / \mathrm{N}$ than high-rep-rate measurements, as $\tau$ increases. This, of course, mirrors the implications of eq 4. This also means that comparing different data acquisition methods should be done as a function of $\tau$.

Figure 4 shows calculated values of CAR vs $\tau$ for a few different experimental configurations. Here, we only consider $\tau$ values larger than the shortest molecular beam pulse, which defines the kinetic resolution (black vertical line). To ensure that the CAR results only from relevant data, the molecular beam repetition rate should be matched to $t_{\min }=5 \tau=1 / f_{\mathrm{MB}}$. This is true for either delay scanning or high-rep-rate detection. This gives rise to CAR plots for optimized delay scanning (blue dashed line) and optimized $1 \mathrm{kHz}$ detection (blue solid line) in Figure 4. The red solid line shows CAR when using optimized $100 \mathrm{kHz}$ detection. We also show in Figure 4 the CAR vs. $\tau$ for unoptimized experiments. Specifically, we show the CAR plot for a delay scan experiment with a fixed $20 \mathrm{~Hz}$ molecular beam (green dashed line) as well as a $1 \mathrm{kHz}$ detection experiment with a $10 \mathrm{~Hz}$ rep-rate molecular beam (magenta solid line).

The range of rates that can be measured with a high $S / N$ is much larger for high-rep-rate detection than for delay scanning. Note that for the optimized experiments, CAR is decreasing with $\tau$; thus, long lifetimes are harder to measure with high $\mathrm{S}$ /

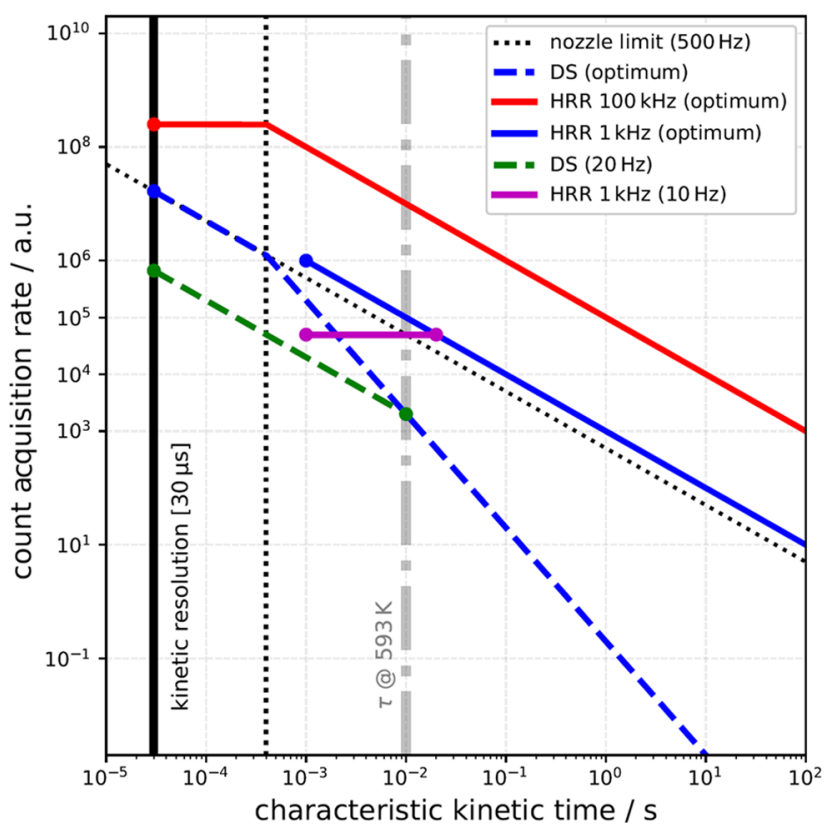

Figure 4. Theoretical count acquisition rates (CAR) vs characteristic kinetic time, $\tau$, for a variety of experimental configurations. Optimized delay scanning (DS, blue dashed line) and $1 \mathrm{kHz}$ high-rep-rate detection (HRR, blue solid line) as well as an optimized high-rep-rate detection with $100 \mathrm{kHz}$ detection (red solid line) experiment are shown-here, only relevant data (see text) is obtained. Experimental configurations presented in this paper are also shown for delay scanning (green dashed line, $\mathrm{CO}$ nozzle at $20 \mathrm{~Hz}$ ) and $1 \mathrm{kHz}$ detection with $\mathrm{CO}$ beam operating at $10 \mathrm{~Hz}$ (magenta solid line). The gray dash-dotted line indicates the value of $\tau$ relevant to our experiments on $\mathrm{CO}$ trapping/desorption, where we measured the improvement to the CAR. The temporal resolution for a transient kinetics experiment is limited by the duration of the molecular beam pulse (black solid line). The minimum time between molecular beam pulses is limited by pumping speed and maximum pulsed valve frequency of $500 \mathrm{~Hz}$ (black dotted line).

$\mathrm{N}$ than are short lifetimes. However, for delay scanning, the CAR is proportional to $\tau^{-2}$ while for high-rep-rate experiments, it is proportional to $\tau^{-1}$. This is reflected in Figure 4 through the slope of CAR vs $\tau$ for delay scan measurements, which is steeper than that of high-rep-rate experiments. Furthermore, increasing $f_{\mathrm{L}}$ further increases CAR. This shows that the highrep-rate method becomes extremely attractive for measuring slow rates. From our experience, the feasibility limit in an optimized delay scanning experiment is reached for $\tau \sim 10-40$ ms. Delay scanning measurements under these conditions take on the order of $1 \mathrm{~h}$. The same limit is reached in a $1 \mathrm{kHz}$ measurement when $\tau \sim 5 \mathrm{~s}$, which can be extended to $500 \mathrm{~s}$ with $100 \mathrm{kHz}$ detection. This shows that the high-rep-rate detection approach can be applied to measure $\tau^{-1}$ values over $\sim 7$ orders of magnitude, whereas delay scanning is limited to at most 3 orders of magnitude. High-rep-rate detection thus enables measurements over a wider temperature range, providing more accurate Arrhenius parameters and greater sensitivity to non-Arrhenius behavior.

We also compare experimentally observed CARs obtained from our actual $\mathrm{CO}$ desorption experiments. The vertical gray dash-dotted line of Figure 4 (marked with $\tau @ 593 \mathrm{~K}$ ) represents the temperature at which the $\mathrm{CO}$ desorption experiments presented in Figure 2 were carried out. Here, delay scanning required $20 \mathrm{~min}$ to obtain $\sim 250$ relevant data, 
while $1 \mathrm{kHz}$ detection provided $\sim 70$ relevant data in $10 \mathrm{~s}$. The derived rate constants were of similar accuracy for both methods. Normalizing to the number of relevant data points obtained, we find that CAR increased by a factor of $\sim 30$ for 1 $\mathrm{kHz}$ detection compared to delay scanning. Seen at the $\tau$-value at $593 \mathrm{~K}$, the theoretical CAR plots (magenta line) and (green dashed line) show a theoretical enhancement factor that is close to the observed enhancement.

3.3. Real-Time Titration Experiment for CO Oxidation at $\mathrm{Pd}(332)$. The velocity-resolved kinetics experiment carried out with delay scanning provides time-resolved information by recording a signal arising from two pulses with a variable delay. Such experiments require that the system under study does not change between each pulse pair; however, this requirement is often not fulfilled in surface chemistry. For example, catalysts can become poisoned with use by buildup of carbon ${ }^{14}$ or other trace impurities. Furthermore, the composition of the surface can change under reactive conditions. ${ }^{10}$ This also has an important implication for molecular beam experiments. For example, if we begin with the $\mathrm{Pd}(332)$ crystal used above for $\mathrm{CO}$ desorption, clean it, and start dosing with the $\mathrm{CO}$ and $\mathrm{O}_{2}$ pulses, the concentration of adsorbed oxygen, $\left[\mathrm{O}^{*}\right]$, will change with time in a way that is determined by the competitive kinetics of $\mathrm{O}_{2}$ dissociation and adsorption, $\mathrm{CO}$ adsorption, reaction, and desorption. Thus, $\left[\mathrm{O}^{*}\right]$ is a complex function of the two beam fluxes and the rates of each elementary process.

This has been shown in detail for $\mathrm{CO}$ oxidation on $\mathrm{Pt}(111)$ using delay scanning, ${ }^{6}$ where a steady-state oxygen concentration, $\left[\mathrm{O}^{*}\right]_{\mathrm{SS}}$, is established over a period of few seconds. Velocity-resolved kinetics exploit such steady-state conditions to investigate the reaction rate's dependence on oxygen coverage. Specifically, $\mathrm{CO}$ and $\mathrm{O}_{2}$ pulsed beams run asynchronously at a controlled repetition rate ratio (RRR) to fix $\left[\mathrm{O}^{*}\right]_{S S}$. Each new value of RRR gives a new value of $\left[\mathrm{O}^{*}\right]_{S S}$ that is determined by a titration. The titration involves first saturating the $\mathrm{Pt}(111)$ surface with oxygen by running the $\mathrm{O}_{2}$ beam for several minutes. This is known to produce an $\mathrm{O}^{*}$ coverage of $\left[\mathrm{O}^{*}\right]_{\text {sat }}=0.25$ Monolayer $(\mathrm{ML})$. We then turn off the $\mathrm{O}_{2}$ beam and run many $\mathrm{CO}$ molecular beam pulses while monitoring the $\mathrm{CO}_{2}$ formation rate at a specific time within the kinetic trace, $t_{\mathrm{BL}}$, the beam laser delay time. The $\mathrm{CO}_{2}$ formation rate at the chosen $t_{\mathrm{BL}}$ changes as more $\mathrm{CO}$ pulses react at the surface, eventually going to zero when all of the $\mathrm{O}^{*}$ is removed from the surface. However, from the titration measurement at a single $t_{\mathrm{BL}}$ it is not possible to determine the oxygen coverage. This is because the transient rate of $\mathrm{CO}_{2}$ formation becomes slower as $\mathrm{O}^{*}$ is removed from the surface. By choosing only one specific $t_{\mathrm{BL}}$ we miss the change of the kinetic trace as a function of titration time. To account for the change of the kinetic trace during the titration, measurements are repeated for various $t_{\mathrm{BL}}$, and the titration curves are integrated over $t_{\mathrm{BL}}$. The integral of such titration curves is proportional to the total oxygen coverage on the surface. We compare the integral from oxygen saturated surfaces with those obtained from a steady-state oxygen covered surface to determine the fraction of the total oxygen coverage that remains under steady-state conditions. Clearly, this procedure is not optimal; ideally, one would like to know the kinetic trace at each point in the titration. While this is tremendously tedious and time-consuming to perform with delay scanning, it is easily achieved with high-rep-rate detection.
Figure 5 shows such a measurement carried out on $\operatorname{Pd}(332)$ at $T_{\mathrm{S}}=503 \mathrm{~K}$. Here, we first saturated the surface with oxygen

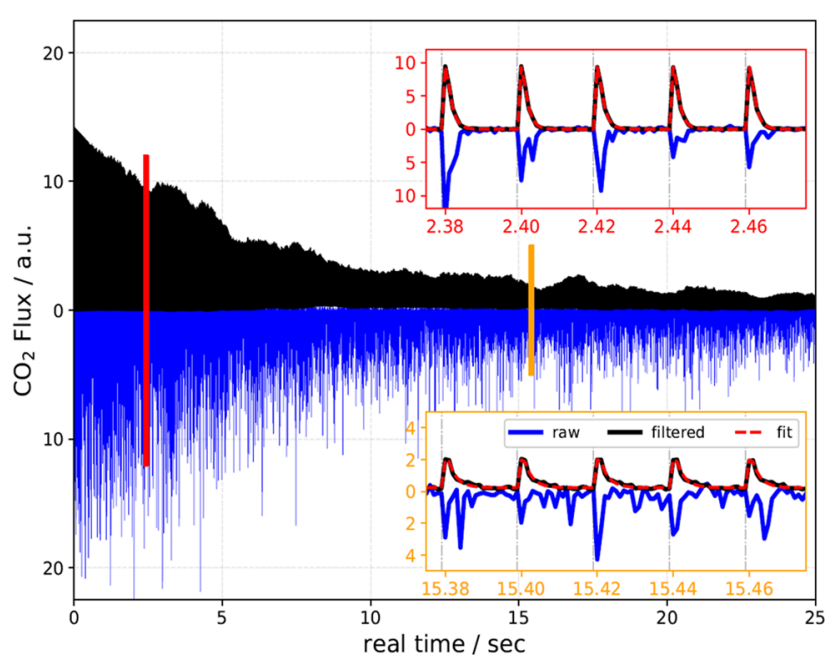

Figure 5. High-rep-rate detection of velocity-resolved kinetics for a nonstationary catalyst. The kinetics of $\mathrm{CO}$ oxidation on $\mathrm{Pd}(332)$ are recorded starting with saturated oxygen coverage. Adsorbed oxygen is removed during the experiment, and the kinetics change accordingly. The surface temperature was $503 \mathrm{~K}$, and the $\mathrm{CO}$ beam operated at 50 $\mathrm{Hz}$. The $\mathrm{CO}$ beam cleans up a preoxidized surface that had been exposed to $300 \pm 80 \mathrm{ML}$ of $\mathrm{O}_{2}$. The raw data are shown as blue lines, the Savitzky-Golay filtered data are shown as black lines. Kinetic fits (first-order decay convoluted with incident beam shape) are shown as red dashed lines in the insets. The insets are indicated by colored bars and borders. The gray dash-dotted line in the insets indicates the reaction time at which the reaction is initiated by the pulsed $\mathrm{CO}$ beam.

by dosing with a $500 \mathrm{~Hz} \mathrm{O}$ molecular beam pulse for $5 \mathrm{~min}$ (total exposure, $300 \pm 80 \mathrm{ML}$ ). The flux of the $\mathrm{O}_{2}$ molecular beam operating at $500 \mathrm{~Hz}$ is $1.0 \pm 0.3 \frac{\mathrm{ML}}{\mathrm{s}}$ with $\approx 2 \times 10^{-3} \frac{\mathrm{ML}}{\text { pulse }}$ The high-rep-rate raw data (blue lines) results from a $\mathrm{CO}$ pulsed beam operating at $50 \mathrm{~Hz}$. The $\mathrm{CO}$ molecular beam operating at $50 \mathrm{~Hz}$ provides a flux of $(6 \pm 2) \times 10^{-2} \mathrm{ML} / \mathrm{s}$ with $\approx 1 \times 10^{-3} \frac{\mathrm{ML}}{\text { pulse }}$ With each CO titrant pulse, a certain amount of oxygen is removed from the surface so that each kinetic trace probes a different $\mathrm{O}$-atom surface coverage. Using a 51-point periodic Savitzky-Golay filter, as described above, we filtered the raw data (blue lines of Figure 5) to yield the filtered data (black lines of Figure 5). The insets in Figure 5 show representative kinetic traces at early and late times in the titration. We find that the signal amplitude decreases and the rate slows with increasing titration time, reflecting the consumption of oxygen with each subsequent $\mathrm{CO}$ pulse. The filtered data can be represented by a first-order decay for the entire $30 \mathrm{~s}$ titration time and for temperatures between 473 and $533 \mathrm{~K}$.

3.4. Reaction Rate Analysis at High Oxygen Coverages. This approach provides new information about the nature of the kinetics and improves the performance of the velocity-resolved kinetics methods. One advantage is the ability to obtain rates of reactions at saturated oxygen coverage, where the absolute oxygen coverage is unambiguously defined. To demonstrate this, we apply a simple model previously suggested by Engel and Ertl to describe $\mathrm{CO}$ oxidation kinetics on Pd. ${ }^{18}$ The model incorporates four processes 


$$
\begin{aligned}
& \mathrm{F}_{t}\left(\mathrm{O}_{2, \mathrm{~g}}\right) \stackrel{S_{\mathrm{O}_{2}}}{\longrightarrow} 2 \mathrm{O}^{*} \\
& \mathrm{~F}_{t}\left(\mathrm{CO}_{\mathrm{g}}\right) \stackrel{S_{\mathrm{CO}}}{\longrightarrow} \mathrm{CO}^{*} \\
& \mathrm{CO}^{*} \stackrel{k_{\mathrm{d}}}{\longrightarrow} \mathrm{CO}_{\mathrm{g}}+* \\
& \mathrm{CO}^{*}+\mathrm{O}^{*} \stackrel{k_{\mathrm{r}}}{\longrightarrow} \mathrm{CO}_{2, \mathrm{~g}}+2^{*}
\end{aligned}
$$

where $\mathrm{X}^{*}$ indicates an adsorbed species $\mathrm{X}$ to the surface, * indicates a free adsorption site, and $F_{t}$ is the time-dependent flux provided by the molecular beams to the surface. $S_{\mathrm{X}}$ is the sticking coefficient of the species $\mathrm{X}$. Under conditions of excess oxygen, the effective first-order rate constant, $k_{\text {eff }}$ is given by

$$
k_{\text {eff }}=k_{\mathrm{r}}\left[\mathrm{O}^{*}\right]+k_{\mathrm{d}}
$$

and the $\mathrm{CO}_{2}$ formation rate is given by

$$
\frac{\mathrm{d}\left[\mathrm{CO}_{2, \mathrm{~g}}\right]}{\mathrm{d} t}=k_{\mathrm{r}}\left[\mathrm{O}^{*}\right]\left[\mathrm{CO}^{*}\right]
$$

We take advantage of the fact that the velocity-resolved kinetics signal is directly proportional to the rate of $\mathrm{CO}_{2, \mathrm{~g}}$ formation and that during the first few $\mathrm{CO}$ pulses arriving at the surface, we probe a well-defined oxygen coverage $[\mathrm{O} *]_{\text {sat }}=$ $0.292 \mathrm{ML}^{6}$ We present examples of such kinetic traces in Figure 6, averaged over the first $20 \mathrm{CO}$ pulses for three values of $T_{S}$. We fit each trace to obtain a first-order time constant $\left(\tau_{\text {eff }}=k_{\text {eff }}^{-1}\right)$. Since $k_{\mathrm{d}}$ is known-in fact, under these conditions, desorption is not competitive and $k_{\text {eff }}=k_{\mathrm{r}}[\mathrm{O} *]_{\text {sat }}$; see the blue shaded area of Figure $3-$ and coverage $\left[\mathrm{O}^{*}\right] \equiv\left[\mathrm{O}^{*}\right]_{\text {sat }}$, we can
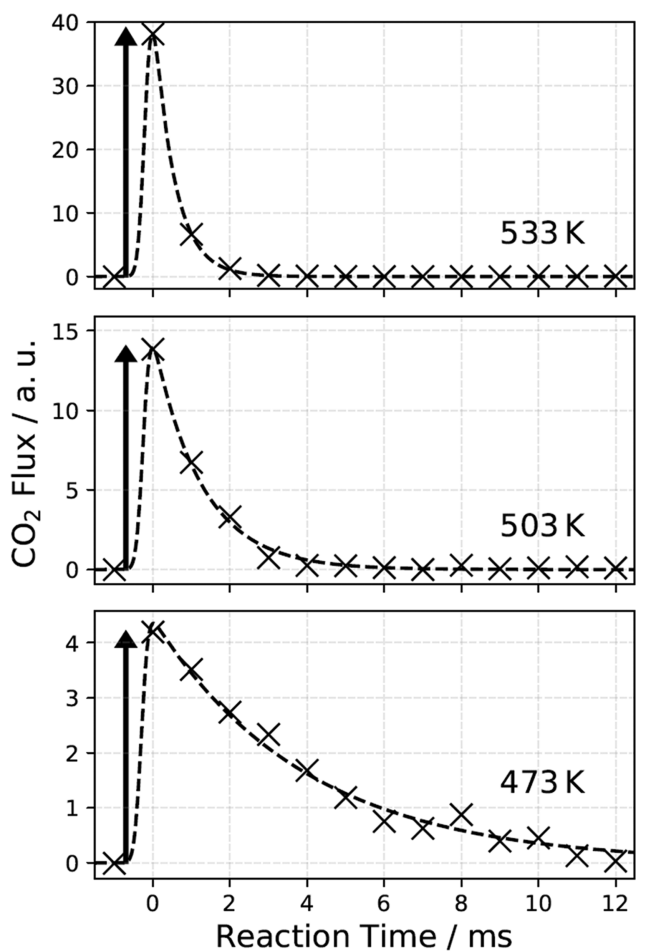

Figure 6. $\mathrm{CO}$ oxidation kinetics at saturated oxygen coverage. Kinetic traces (crosses) obtained by averaging over the first 20 pulses in experiments like those of Figure 5. The dashed lines are fits to a firstorder decay (convoluted over the incident beam). The arrows indicate the relative initial rates in the three experiments. easily determine $k_{\mathrm{r}}$ at all three values of $T_{\mathrm{S}}$. The derived effective reaction rate constants $\left(k_{\mathrm{r}}\left[\mathrm{O}^{*}\right]_{\text {sat }}\right)$ are shown in Figure 7 as an Arrhenius plot. Note that in Figure 6 for $T_{\mathrm{S}}=$

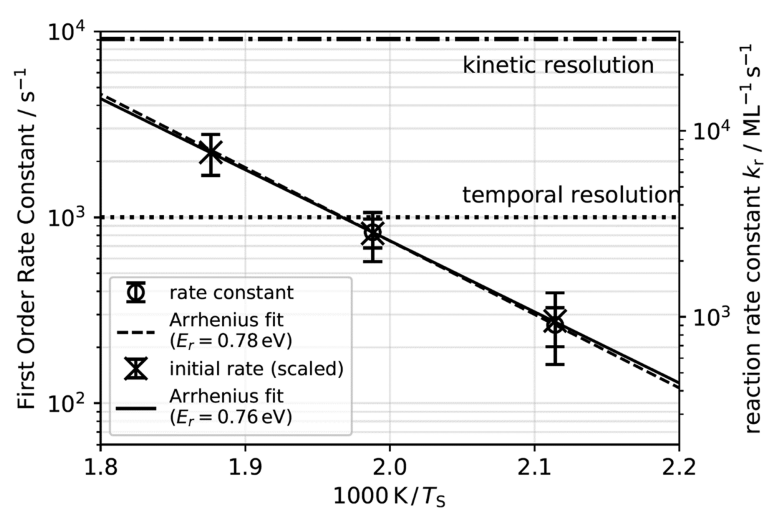

Figure 7. Temperature dependence of $\mathrm{CO}$ oxidation rate constants at saturated oxygen coverage. The first-order rate constants for $\mathrm{CO}_{2}$ formation determined from the data of Figure 6. The circles are the rate constants determined from the shape of the single-pulse kinetic trace, and the crosses are initial rates determined from their amplitude. The initial rates are scaled to match the first-order rate constants at low temperatures. The dotted line is the limit above which the rate constants cannot be derived from the shape of the kinetic trace. The dash-dotted line is the kinetic resolution (in this experiment, around $110 \mu \mathrm{s}$ ) above which no kinetic information can be derived from transient kinetics. The full and dashed curves are Arrhenius fits to the initial rate and the first-order rate constant, respectively.

$533 \mathrm{~K}$, the temporal resolution is insufficient to provide a reliable fit. This problem could be solved by repetitive measurements of this type at a variety of delays of the $\mathrm{CO}$ molecular beam pulse-interleaved in $0.1 \mathrm{~ms}$ steps. One could also use a higher repetition rate laser. Alternatively, $k_{\mathrm{r}}$ can be obtained simply from the amplitude of the kinetic trace, shown as arrows in Figure 6, which is proportional to the initial rate of product formation. This allows the rate constants at all three temperatures to be placed on the same scale using the Arrhenius law. In Figure 7, the values derived from initial rates $(x)$ are placed on an absolute scale in comparison to the rate constants obtained by exponential fitting at the lower two temperatures (o). The best-fit Arrhenius parameters for the reaction rate constant $k_{\mathrm{r}}$ are $E_{\mathrm{a}}=0.76 \pm 0.02 \mathrm{eV}$ and $A=$ $10^{11.0 \pm 0.4} \mathrm{~s}^{-1} \mathrm{ML}^{-1}$. These results are consistent with independently obtained results using delay scanning.

3.5. Diffusion-Limited Surface Reaction Rates. In the course of studying the behavior of high-rep-rate detection, we made what were, at first, surprising observations. We found that near the end of titrations when the rate of $\mathrm{CO}_{2}$ formation had nearly vanished, oxygen coverage remained on the surface in regions outside the crossing region of the two molecular beams. By translating the crystal in a direction perpendicular to the surface normal, we could observe a sudden increase of the $\mathrm{CO}_{2}$ production rate. These observations indicated that a successful modeling of these experiments would require characterizing both the spatial and temporal evolution of the reactant coverages.

In this section, we describe such modeling showing that titration experiments often produce conditions where $\mathrm{CO}$ diffusion affects the rates of reactions. 
We first imagine dividing the reacting surface into $j$ spatial elements. The concentrations of $\mathrm{CO}^{*}$ and $\mathrm{O}^{*}$ in spatial element $j$, defined as $\left[\mathrm{O}^{*}\right]_{j}$ and $\left[\mathrm{CO}^{*}\right]_{j}$, are given by reactive terms

$$
\begin{aligned}
& \left(\frac{\mathrm{d}[\mathrm{CO} *]}{\mathrm{d} t}\right)_{j}^{\mathrm{rct}}=S_{\mathrm{CO}, j} F_{t, j}\left(\mathrm{CO}_{\mathrm{g}}\right)-k_{\mathrm{d}}\left[\mathrm{CO}^{*}\right]_{j}-k_{\mathrm{r}}\left[\mathrm{O}^{*}\right]_{j} \\
& {\left[\mathrm{CO}^{*}\right]_{j}} \\
& \left(\frac{\mathrm{d}[\mathrm{O} *]}{\mathrm{d} t}\right)_{j}^{\mathrm{rct}}=2 S_{\mathrm{O}_{2}, j} F_{t, j}\left(\mathrm{O}_{2, \mathrm{~g}}\right)-k_{\mathrm{r}}\left[\mathrm{O}^{*}\right]_{j}\left[\mathrm{CO}^{*}\right]_{j}
\end{aligned}
$$

and diffusive terms

$$
\begin{aligned}
& \left(\frac{\mathrm{d}\left[\mathrm{CO}^{*}\right]}{\mathrm{d} t}\right)_{j}^{\mathrm{dif}}=D_{\mathrm{CO}, j} \Delta\left[\mathrm{CO}^{*}\right]_{j} \\
& \left(\frac{\mathrm{d}[\mathrm{O} *]}{\mathrm{d} t}\right)_{j}^{\mathrm{dif}}=D_{\mathrm{O}, j} \Delta\left[\mathrm{O}^{*}\right]_{j}
\end{aligned}
$$

The total rate is the sum of reactive (eqs 14 and 15) and diffusive (eqs 16 and 17) contributions. In eqs 16 and 17, $D_{n}$ and $\Delta\left[n^{*}\right]_{j}$ are the species-specific and concentrationindependent diffusion coefficients and Laplacian of the concentration, respectively, used in application of Fick's second law of diffusion, and $F_{t, j}$ is the time-dependent incoming flux to the spatial element $j$, produced by the molecular beams.

The dosing function $F_{t, j}$ is described with a periodic function (in time) that reassembles the spatial, $g\left(r_{j}\right)$, and temporal, $f(t)$, shapes of our molecular beams. Specifically, we modeled it with

$$
f(t)=\cos ^{2 n}\left(\pi \operatorname{RR}\left(t-t_{0}\right)\right)
$$

where $R R$ is the repetition rate of the nozzle, $t_{0}$ is the reference timing, and $n$ is an integer chosen to best represent the temporal shape of the beam. Using ion imaging, we experimentally determined the spatial intensity profile of each molecular beam, from which we deduced their radial profiles. Both molecular beams have a nominal projected diameter of $2 \mathrm{~mm}$. For $F_{t, j}$, we use a flattop Gaussian that resembles the experimentally determined radial profile, $g\left(r_{j}\right)$, of the beam, which is given by

$$
g\left(r_{j}\right)=\exp \left(-\left[\frac{r_{j}^{2}}{2 \sigma^{2}}\right]^{m}\right)
$$

where $m$ and $\sigma$ are the parameters representing the shape of the experimental beam profile. The combined and normalized dosing function is then given by

$$
F_{t, j}=\frac{g\left(r_{j}\right) f(t)}{N}
$$

where $N$ is the normalization to define the observed molecular beam flux.

We made sure that both molecular beams overlap on the surface and checked this by ensuring that oxygen coverage remained symmetrically distributed around the molecular beams crossing point at the end of the titration measurement. Hence, we conclude that our experiments approximately preserve radial symmetry, which allows us to solve the diffusion equations, eqs 16 and 17, in polar coordinates. The diffusion formalism is derived in the Supporting Information (SI). The rate equations including diffusion and reaction are solved numerically using LSODA from the Fortran ODEPACK library. ${ }^{19}$ The concentrations of $\mathrm{CO}^{*}$ and $\mathrm{O}^{*}$ in each spatial element $j$ are propagated in time.

To simulate measurements like those of Figure 5, we initiate the model calculations with adsorbed oxygen produced by many pulses of the $\mathrm{O}_{2}$ beam. This requires an initial $\mathrm{O}^{*}$ spatial profile (black line in Figure 8 ) that is much broader than the

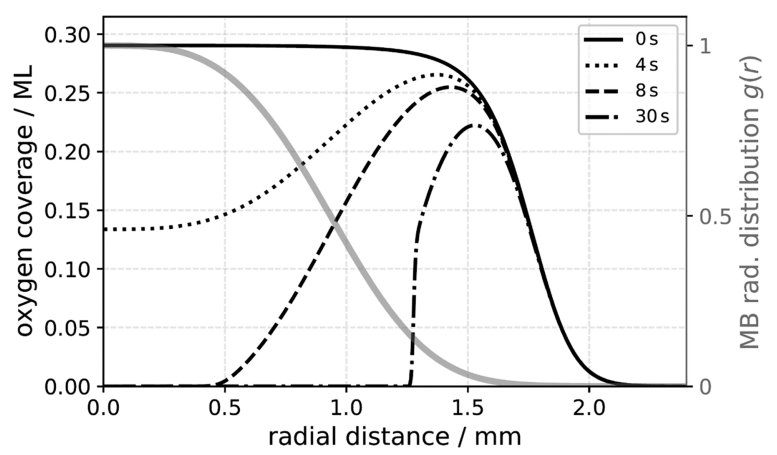

Figure 8. Spatial distributions of adsorbed oxygen atoms during a $\mathrm{CO}$ oxidation titration. The distributions are assumed cylindrically symmetrical about the $\mathrm{CO}$ beam axis. The radial distance from the CO beam center line is shown on the $x$-axis. The solid black line indicates the initial oxygen coverage distribution produced by long exposure with a molecular beam of $\mathrm{O}_{2}$. The radial distribution of the $\mathrm{CO}$ beam (gray thick line) peaks near 0 and preferentially removes $\mathrm{O}$ atoms there. As time progresses, a "doughnut hole" reaction develops, where the $\mathrm{CO}$ is concentrated along the $\mathrm{CO}$ beam center line and adsorbed oxygen atoms form a ring around the $\mathrm{CO}$ beam. In later stages of titration, the reaction forms a front where the $\mathrm{CO}$ and $\mathrm{O}$ concentrations overlap. Diffusion of $\mathrm{CO}$ from the center of the doughnut hole to the oxygen ring also influences the reaction rate.

nominal $\mathrm{O}_{2}$ beam profile (thick gray line in Figure 8), as $\mathrm{O}^{*}$ coverage quickly saturates near the center of the beam, and after that, only the wings of the $\mathrm{O}_{2}$ beam add additional $\mathrm{O} *$. We simulated the spatial evolution of concentrations within a radial extent of $3 \mathrm{~mm}$ and with each spatial element, $j$, being 5 $\mu \mathrm{m}$ in size. The corresponding total $\mathrm{CO}_{2}$ formation rate is given by summing the rate of each spatial element $j$ and weighting it by the respective area $A_{j}$, in the following manner

$$
\frac{\mathrm{d}\left[\mathrm{CO}_{2}\right]_{t}}{\mathrm{~d} t}=k_{\mathrm{r}} \sum_{j=0}^{j_{\max }}\left[\mathrm{O}^{*}\right]_{t, j}\left[\mathrm{CO}^{*}\right]_{t, j} A_{j}
$$

where the area of the $j$ th spatial element is given by

$$
A_{j}=\pi(2 j+1) l_{0}^{2}
$$

The simulation accounts for the influence of reactions $8-11$ as well as $\mathrm{CO}$ diffusion. The reaction rate constants were determined previously (see Sections 3.1 and 3.3). Oxygen desorption is unimportant at these surface temperatures. ${ }^{20,21}$ Oxygen diffusion is found to be unimportant under our conditions. ${ }^{22}$ We estimated the diffusion coefficient for $\mathrm{CO}$ using an activation energy of $0.12 \mathrm{eV}$ from ref 23, and the fitted prefactor for $\mathrm{CO}$ diffusion needed to obtain agreement with our measurements. The optimized prefactor was $10^{-3.7 \pm 0.3} \mathrm{~cm}^{2}$ $\mathrm{s}^{-1}$. The CO diffusion rates we obtain in this way are 

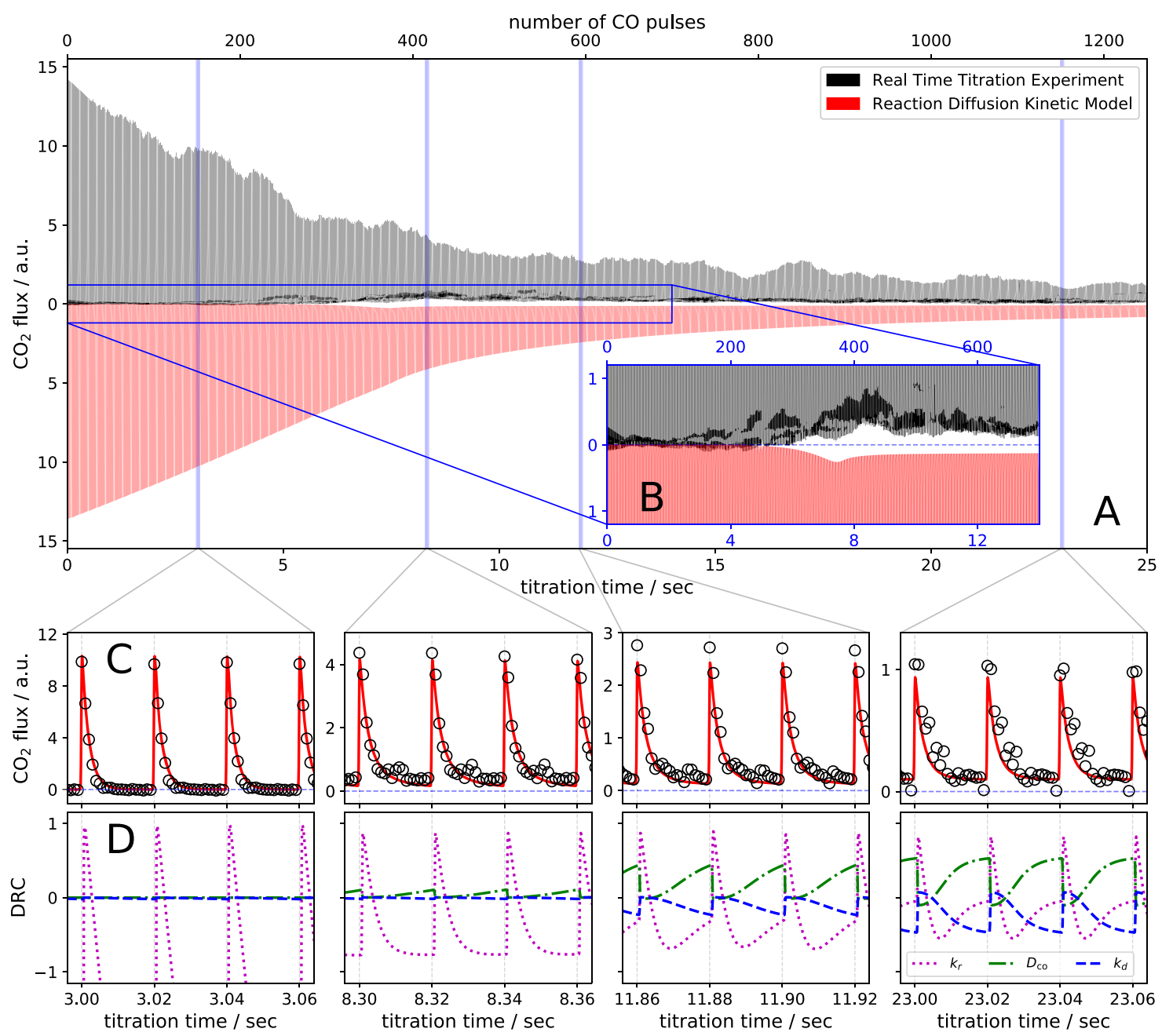

Figure 9. Comparison of the model of real-time titration (red solid lines) with measurements (black solid lines and open circles). The onset of the diffusion-controlled regime is indicated in (B), where a continuous $\mathrm{CO}_{2}$ production rate forms. (C) Results at 3.0, 8.3, 11.9, and 23.0 s after the start of the titration. The degree of rate control (DRC) is shown in (D) for three elementary processes: CO oxidation reaction (magenta dotted line), $\mathrm{CO}$ desorption (blue dashed line), and CO diffusion (green dash-dotted line). The DRC for O diffusion is for all conditions at least 2 orders of magnitude smaller and is therefore not shown. See text. Experimental conditions are as stated in Figure 5.

consistent with previous measurements on $\mathrm{Pd}(111)$ - see the SI.

We derived the absolute incident beam fluxes from measurements of the steady-state pressures of $\mathrm{CO}$ and $\mathrm{O}_{2}$ in the UHV chamber combined with a knowledge of the chamber pumping speed. The model results were insensitive to the $\mathrm{O}_{2}$ flux, but highly sensitive to the assumed $\mathrm{CO}$ flux. We found best agreement with experiment when using a CO flux 30\% smaller than that derived from our experimental estimate.

We used a second-order Langmuir expression for the coverage-dependent sticking coefficient of $\mathrm{O}_{2}$,

$$
S_{\mathrm{O}_{2}}([\mathrm{O} *])=S_{\mathrm{O}_{2}, 0}\left(1-\frac{[\mathrm{O} *]}{\left[\mathrm{O}^{*}\right]_{\max }}\right)^{2}
$$

with $S_{\mathrm{O}_{2}, 0}=0.4 .{ }^{16}$ Best agreement with the experiment is achieved when an oxygen coverage-independent sticking coefficient of $0.6 \pm 0.1$ is used for CO. We assume that the sticking probability of $\mathrm{CO}$ decreases linearly with $\mathrm{CO}$ coverage.

Figure 9A shows a comparison of this model to the titration experiment of Figure 5. Note that the amplitude quickly decays over the first 300-400 CO beam pulses, thereafter decaying more slowly, a behavior that is captured in the kinetic model. The transition between the fast and slow decay regions is accompanied by an increase of the baseline (shown in magnification in Figure 9B). This indicates a continuous production of $\mathrm{CO}_{2}$. The experimentally observed increase of the baseline is also present in the model. Looking in more detail (Figure 9C), we find that the single-pulse transient rate is decreasing with increasing titration time; furthermore, the transient rates are well reproduced by the kinetic model as is the continuous production of $\mathrm{CO}_{2}$ seen in the later stages of the titration.

The qualitative behavior can be understood by recalling that the amplitude of the titration curve reflects the initial rate of $\mathrm{CO}_{2}$ production, which is directly proportional to the oxygen coverage. With increasing titration time, the initial rate decreases, indicating that the oxygen coverage is dropping. As a consequence of the reduced reaction rate at a lower oxygen coverage, the lifetime of $\mathrm{CO}$ molecules on the surface increases, while the rate of $\mathrm{CO}$ adsorption remains constant. Since CO's desorption rate is slow at these temperatures, $\mathrm{CO}$ begins to build up from one molecular beam pulse to the next; 
this leads to quasi-continuous $\mathrm{CO}_{2}$ formation and to baseline increase at later times in the titration.

We also performed a sensitivity analysis of the fit to the titration kinetics. The degree of rate control ${ }^{24}$ exhibited by the kinetic parameter, $k_{i}$ is given by a sensitivity coefficient, $X_{i, t}$

$$
X_{i, t}=\frac{k_{i}}{R_{t}}\left(\frac{\mathrm{d} R_{t}}{\mathrm{~d} k_{i}}\right)_{k_{j \neq i}}
$$

where $R_{t}$ is the $\mathrm{CO}_{2}$ formation rate. A high absolute value of $X_{i, t}$ indicates the importance of the process to the reaction rate. A positive (negative) value of $X_{i, t}$ means that an increase of the rate parameter produces an increase (decrease) of the $\mathrm{CO}_{2}$ formation rate. In Figure 9D, we plot $X_{i, t}$ for reaction (purple, dotted), CO desorption (blue, dashed), and $\mathrm{CO}$ diffusion (green, dash-dotted).

The reaction between $\mathrm{CO}^{*}$ and $\mathrm{O}^{*}$ dominates the rate of product formation up to a titration time of about $7 \mathrm{~s}$; thereafter, $\mathrm{CO}$ desorption and diffusion become increasingly important. Between 12 and 24 s, where the three processes are of similar importance, their influence appears at different points in the kinetic trace. Consider the kinetic traces found at $\sim 23 \mathrm{~s}$. Here, the beginning of the kinetic trace is dominated by the influence of the reaction, whereas diffusion and desorption influence later times in the trace. Note that desorption decreases while diffusion increases the rate of $\mathrm{CO}_{2}$ production. This can be understood by realizing that at later stages of the titration, $\mathrm{O}^{*}$ has been depleted near the center of the $\mathrm{CO}$ beam. Each new $\mathrm{CO}$ pulse produces a higher $\mathrm{CO}^{*}$ concentration in the doughnut hole of $\mathrm{O}^{*}$ concentration (see Figure 8). These are the conditions where the quasicontinuous $\mathrm{CO}_{2}$ formation rate (i.e., the $\mathrm{CO}_{2}$ being produced prior to the next pulse) can appear as it is due to a diffusioncontrolled reaction between $\mathrm{CO}^{*}$ and $\mathrm{O}^{*}$.

Figure 8 shows the model's predictions of the oxygen's spatial distribution at various times during the titration. As explained above, the initial oxygen coverage distribution (black solid line) on the surface is broader than the $\mathrm{CO}$ or $\mathrm{O}_{2}$ beam's spatial profiles (gray solid line) used to dose the surface. At the early stages of titration ( $4 \mathrm{~s}$-dotted black line), CO flux is highest near the beam center line where $\mathrm{O}^{*}$ removal proceeds most rapidly. At $8 \mathrm{~s}$ (dashed black line), oxygen is removed near the center of the $\mathrm{CO}$ beam. In the central region of the spatial distribution, where $\mathrm{O}^{*}$ has now been depleted, CO's lifetime increases and begins building up from pulse to pulse. Hence, a spatially inhomogeneous "doughnut" reaction is produced with high $\mathrm{CO}$ coverage near the center of the beam and high $\mathrm{O}$-coverage near the wings of the beam (see the $\mathrm{SI}$ ). The quasi-continuous $\mathrm{CO}_{2}$ formation is produced at the intersection of the $\mathrm{CO}^{*}$ - and $\mathrm{O}^{*}$-rich regions, forming a reaction front. The product formation rate at the reaction front depends not only on the reaction rate constant but also on the $\mathrm{CO}$ diffusion coefficient. While the stationary $\mathrm{CO}_{2}$ formation is from a diffusion-controlled reaction, the transient rate induced by a $\mathrm{CO}$ pulse at late titration times is due to direct population of oxygen-rich regions from the outer flanks of the $\mathrm{CO}$ beam and is only slightly influenced by the mobility of the reactants.

With our validated kinetic model, we can also estimate the associated reaction front speed (see the SI), which is a characteristic property that can be measured for spatiotemporal pattern formation. In Figure 10, the reaction front speed at $503 \mathrm{~K}$ is shown as a function of titration time. Prior to $6 \mathrm{~s}$ after the beginning of the titration, no reaction front is formed.

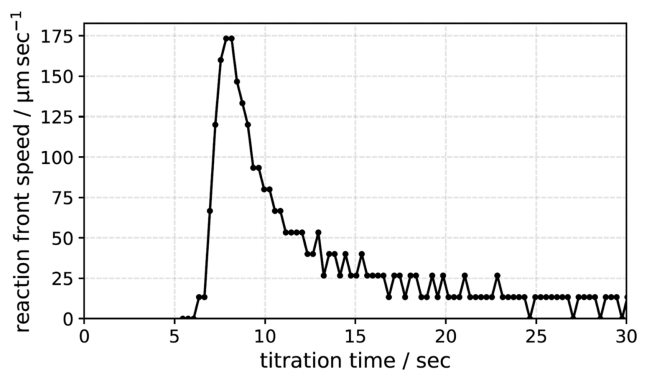

Figure 10. Model's prediction of the reaction front speed as a function of titration time at a surface temperature of $503 \mathrm{~K}$.

However, from 6 to $8 \mathrm{~s}$ titration time, a reaction front forms and its speed accelerated to $175 \mu \mathrm{m} / \mathrm{s}$. With increasing titration time, the front speed decreases, reaching speeds of around $10 \mu \mathrm{m} / \mathrm{s}$ at titration times longer than $15 \mathrm{~s}$. We emphasize that the derived values of front speed are similar to those obtained in previous work for $\mathrm{CO}$ oxidation on $\mathrm{Pt}(110)$, which ranged from 1 to $100 \mu \mathrm{m} / \mathrm{s}^{25,26}$ The fact that we derive front speeds nearly a factor of 2 higher than that work probably results from faster thermal diffusion for $\mathrm{CO}$ on $\mathrm{Pd}$ compared to Pt. $^{23,27}$

It is important to highlight that we have modeled the realtime titration experiment without coverage-dependent rate constants. Since we achieve good correspondence with the experiment, we claim that the rate constants have weak dependence on oxygen coverage in $\mathrm{CO}$ oxidation on $\mathrm{Pd}(332)$. However, this is in contradiction to the findings that were previously made on $\operatorname{Pd}(111)$ by Engel and Ertl. ${ }^{18}$ We also find that our reaction rate constant is about 4-8 times higher than those reported from $\mathrm{Pd}(111)$. We think that steps lead to a higher reaction rate, consistent with previous observations on $\mathrm{Pd}^{28}$ and $\mathrm{Pt}^{6}$ The reason why we have not taken reaction at steps and terraces explicitly into account is that we have not needed it for good match with the experiment. This is probably due to a rather fast exchange of $\mathrm{CO}$ and $\mathrm{O}$ atoms between terraces and steps, which leads to an effective reaction rate composed of both reactions at steps and terraces. We plan to investigate the details of the kinetic mechanism of $\mathrm{CO}$ oxidation at steps and terraces of Pd further in future.

\section{CONCLUSIONS}

This work shows how high-repetition-rate lasers and ion imaging detection can be used to obtain the kinetic traces of catalytic processes from a single molecular beam pulse, overcoming the need for delay scans that are typical for pump-probe methods. The new approach provides an increased duty cycle, resulting in rates of acquisition for kinetic data that are 10-1000 times faster than conventional delay scanning methods. The new method can measure rates over 5-7 orders of magnitude, dramatically better than when using delay scanning. The method is particularly attractive for measuring slow processes where temporal dilution would make delay scanning impossible.

This new approach can also be used to study catalytic reaction rates under conditions where the catalyst composition is changing under reactive conditions. We demonstrated this with a real-time titration experiment, where the transient $\mathrm{CO}_{2}$ production rates were obtained at many points in a $\mathrm{CO}$ oxidation titration experiment. Specifically, we showed that the transient rate of $\mathrm{CO}_{2}$ formation could be measured from each subsequent $\mathrm{CO}$ pulse, where each pulse probes a different $\mathrm{O}$ - 
atom coverage on the surface. From accurate modeling of the titration experiment, we are able to derive various rate constants relevant to $\mathrm{CO}$ oxidation on $\mathrm{Pd}(332)$. Of particular novelty, we easily found conditions where the $\mathrm{CO}$ oxidation was diffusion-controlled. The constants obtained in this work for $\mathrm{CO}$ oxidation reaction, $\mathrm{CO}$ desorption, and $\mathrm{CO}$ diffusion, respectively, are summarized here

$$
\begin{aligned}
& \frac{k_{\mathrm{r}}(T)}{\mathrm{ML}^{-1} \mathrm{~s}^{-1}}=10^{11.0 \pm 0.4} \exp \left(-\frac{0.76 \pm 0.02 \mathrm{eV}}{k_{\mathrm{B}} T}\right) \\
& \frac{k_{\mathrm{d}}(T)}{\mathrm{s}^{-1}}=10^{15.6 \pm 0.3} \exp \left(-\frac{1.58 \pm 0.02 \mathrm{eV}}{k_{\mathrm{B}} T}\right) \\
& \frac{D_{\mathrm{CO}}(T)}{\mathrm{cm}^{2} \mathrm{~s}^{-1}}=10^{-3.7 \pm 0.3} \exp \left(-\frac{0.12 \mathrm{eV}}{k_{\mathrm{B}} T}\right)
\end{aligned}
$$

Our results are also consistent with an oxygen-coverageindependent sticking coefficient of $\mathrm{CO}$ of $0.6 \pm 0.1$. The desorption and diffusion rate constants of $\mathrm{CO}$ agree well with the parameters determined earlier from $\operatorname{Pd}(111)$, indicating that $\mathrm{CO}$ has no energetic preference for steps and that they are not influencing its mobility on the surface. The reaction rate constant is found to be approximately a factor $4-8$ higher than previous reports for $\mathrm{Pd}(111)$, indicating that steps are more reactive for $\mathrm{CO}$ oxidation on $\mathrm{Pd}$ than terraces.

While in this work we were limited to a detection rep-rate of $1 \mathrm{kHz}$ due to the fact that we used a Ti:sapphire laser, we plan to extend our capabilities to a detection rate of $100 \mathrm{kHz}$ and study reaction rates at changing catalyst conditions in more detail using a $\mathrm{Yb}$-Fiber laser. We think that this method offers the possibility to accurately study catalytic reaction rates and kinetic mechanisms at the intersection between the welldefined conditions that are desirable for surface science and the more dynamic conditions relevant to industrial catalysis.

\section{ASSOCIATED CONTENT}

\section{SI Supporting Information}

The Supporting Information is available free of charge at https://pubs.acs.org/doi/10.1021/acscatal.0c03773.

Diffusion equations for simulation of titrations, comparison of $\mathrm{CO}$ diffusion rates to previous reports, and details on the determination of reaction front speeds (PDF)

\section{AUTHOR INFORMATION}

\section{Corresponding Authors}

Theofanis Kitsopoulos - Institute for Physical Chemistry, Georg-August University of Goettingen, 37077 Goettingen, Germany; Department of Dynamics at Surfaces, Max Planck Institute for Biophysical Chemistry, 37077 Goettingen, Germany; Department of Chemistry, University of Crete, 70013 Heraklion, Greece; Institute of Electronic Structure and Lasen冈FORTH, 70013 Heraklion, Greece; orcid.org/ 0000-0001-6228-1002; Email: theo.kitsopoulos@ mpibpc.mpg.de

Alec M. Wodtke - Institute for Physical Chemistry and International Center for Advanced Studies of Energy Conversion, Georg-August University of Goettingen, 37077 Goettingen, Germany; Department of Dynamics at Surfaces, Max Planck Institute for Biophysical Chemistry, 37077
Goettingen, Germany; 다이이.org/0000-0002-6509-2183; Email: alec.wodtke@mpibpc.mpg.de

\section{Authors}

Dmitriy Borodin - Institute for Physical Chemistry, GeorgAugust University of Goettingen, 37077 Goettingen, Germany; Department of Dynamics at Surfaces, Max Planck Institute for Biophysical Chemistry, 37077 Goettingen, Germany

Kai Golibrzuch - Department of Dynamics at Surfaces, Max Planck Institute for Biophysical Chemistry, 37077 Goettingen, Germany

Michael Schwarzer - Institute for Physical Chemistry, GeorgAugust University of Goettingen, 37077 Goettingen, Germany

Jan Fingerhut - Institute for Physical Chemistry, GeorgAugust University of Goettingen, 37077 Goettingen, Germany

Georgios Skoulatakis - Department of Dynamics at Surfaces, Max Planck Institute for Biophysical Chemistry, 37077 Goettingen, Germany

Dirk Schwarzer - Department of Dynamics at Surfaces, Max Planck Institute for Biophysical Chemistry, 37077

Goettingen, Germany; 이이이.org/0000-0003-3838-2211

Thomas Seelemann - LaVision GmbH, 37081 Goettingen, Germany

Complete contact information is available at: https://pubs.acs.org/10.1021/acscatal.0c03773

\section{Funding}

D.B. thanks the BENCh graduate school, funded by the DFG (389479699/ GRK2455). T.K. and A.M.W. acknowledge the European Research Council (ERC) under the European Union's Horizon 2020 research and innovation program under grant agreements 833404 and 742422 , respectively.

\section{Notes}

The authors declare no competing financial interest.

\section{REFERENCES}

(1) Schwarz, J. A.; Madix, R. J. Modulated beam relaxation spectrometry. Surf. Sci. 1974, 46, 317-341.

(2) Gland, J. L.; Kollin, E. B. Carbon monoxide oxidation on the $\mathrm{Pt}(111)$ surface: Temperature programmed reaction of coadsorbed atomic oxygen and carbon monoxide. J. Chem. Phys. 1983, 78, 963974.

(3) Brown, L. S.; Sibener, S. J. A molecular beam scattering investigation of the oxidation of $\mathrm{CO}$ on $\mathrm{Rh}(111)$. I. Kinetics and mechanism. J. Chem. Phys. 1988, 89, 1163-1169.

(4) Zaera, F. Use of molecular beams for kinetic measurements of chemical reactions on solid surfaces. Surf. Sci. Rep. 2017, 72, 59-104.

(5) Golibrzuch, K.; Shirhatti, P. R.; Geweke, J.; Werdecker, J.; Kandratsenka, A.; Auerbach, D. J.; Wodtke, A. M.; Bartels, C. CO desorption from a catalytic surface: elucidation of the role of steps by velocity-selected residence time measurements. J. Am. Chem. Soc. 2015, 137, 1465-1475.

(6) Neugebohren, J.; Borodin, D.; Hahn, H. W.; Altschaffel, J.; Kandratsenka, A.; Auerbach, D. J.; Campbell, C. T.; Schwarzer, D.; Harding, D. J.; Wodtke, A. M.; Kitsopoulos, T. N. Velocity-resolved kinetics of site-specific carbon monoxide oxidation on platinum surfaces. Nature 2018, 558, 280-283.

(7) Harding, D. J.; Neugebohren, J.; Hahn, H.; Auerbach, D. J.; Kitsopoulos, T. N.; Wodtke, A. M. Ion and velocity map imaging for surface dynamics and kinetics. J. Chem. Phys. 2017, 147, No. 013939.

(8) Harding, D. J.; Neugebohren, J.; Auerbach, D. J.; Kitsopoulos, T. N.; Wodtke, A. M. Using Ion Imaging to Measure Velocity Distributions in Surface Scattering Experiments. J. Phys. Chem. A 2015, 119, 12255-12262. 
(9) Park, G. B.; Kitsopoulos, T. N.; Borodin, D.; Golibrzuch, K.; Neugebohren, J.; Auerbach, D. J.; Campbell, C. T.; Wodtke, A. M. The kinetics of elementary thermal reactions in heterogeneous catalysis. Nat. Rev. Chem. 2019, 3, 723-732.

(10) Kondratenko, E. V.; Ovsitser, O.; Radnik, J.; Schneider, M.; Kraehnert, R.; Dingerdissen, U. Influence of reaction conditions on catalyst composition and selective/non-selective reaction pathways of the ODP reaction over $\mathrm{V} 2 \mathrm{O} 3, \mathrm{VO} 2$ and $\mathrm{V} 2 \mathrm{O} 5$ with $\mathrm{O} 2$ and $\mathrm{N} 2 \mathrm{O}$. Appl. Catal., A 2007, 319, 98-110.

(11) Reuter, K.; Scheffler, M. Composition, structure, and stability ofRuO2(110)as a function of oxygen pressure. Phys. Rev. B 2001, 65, No. 1038.

(12) Reuter, K.; Scheffler, M. First-principles atomistic thermodynamics for oxidation catalysis: surface phase diagrams and catalytically interesting regions. Phys. Rev. Lett. 2003, 90, No. 046103.

(13) Reuter, K.; Scheffler, M. Composition and structure of theRuO2(110)surface in anO2and $\mathrm{CO}$ environment: Implications for the catalytic formation ofCO2. Phys. Rev. B 2003, 68, No. 368.

(14) Beebe, T. P.; Goodman, D. W.; Kay, B. D.; Yates, J. T. Kinetics of the activated dissociative adsorption of methane on the low index planes of nickel single crystal surfaces. J. Chem. Phys. 1987, 87, 23052315.

(15) Savitzky, A.; Golay, M. J. E. Smoothing and Differentiation of Data by Simplified Least Squares Procedures. Anal. Chem. 1964, 36, 1627-1639.

(16) Engel, T. A molecular beam investigation of $\mathrm{He}, \mathrm{CO}$, and $\mathrm{O} 2$ scattering from $\operatorname{Pd}(111)$. J. Chem. Phys. 1978, 69, No. 373.

(17) Conrad, H.; Ertl, G.; Koch, J.; Latta, E. E. Adsorption of CO on Pd single crystal surfaces. Surf. Sci. 1974, 43, 462-480.

(18) Engel, T.; Ertl, G. A molecular beam investigation of the catalytic oxidation of CO on $\operatorname{Pd}(111)$. J. Chem. Phys. 1978, 69, 12671281.

(19) Petzold, L. Automatic Selection of Methods for Solving Stiff and Nonstiff Systems of Ordinary Differential Equations. SIAM J. Sci. Stat. Comput. 1983, 4, 136-148.

(20) Banse, B. A.; Koel, B. E. Interaction of oxygen with Pd(111): High effective $\mathrm{O} 2$ pressure conditions by using nitrogen dioxide. Surf. Sci. 1990, 232, 275-285.

(21) Guo, X.; Hoffman, A.; Yates, J. T. Adsorption kinetics and isotopic equilibration of oxygen adsorbed on the $\operatorname{Pd}(111)$ surface. J. Chem. Phys. 1989, 90, 5787-5792.

(22) Rose, M. K.; Borg, A.; Dunphy, J. C.; Mitsui, T.; Ogletree, D. F.; Salmeron, M. Chemisorption of atomic oxygen on $\operatorname{Pd}(111)$ studied by STM. Surf. Sci. 2004, 561, 69-78.

(23) Mitsui, T.; Rose, M. K.; Fomin, E.; Ogletree, D. F.; Salmeron, M. Diffusion and pair interactions of CO molecules on $\operatorname{Pd}(111)$. Phys. Rev. Lett. 2005, 94, No. 036101.

(24) Campbell, C. T. The Degree of Rate Control: A Powerful Tool for Catalysis Research. ACS Catal. 2017, 7, 2770-2779.

(25) Jakubith, S.; Rotermund, H. H.; Engel, W.; von Oertzen, A.; Ertl, G. Spatiotemporal concentration patterns in a surface reaction: Propagating and standing waves, rotating spirals, and turbulence. Phys. Rev. Lett. 1990, 65, 3013-3016.

(26) Rotermund, H. H.; Jakubith, S.; von Oertzen, A.; Ertl, G. Solitons in a surface reaction. Phys. Rev. Lett. 1991, 66, 3083-3086.

(27) Reutt-Robey, J. E.; Doren, D. J.; Chabal, Y. J.; Christman, S. B. $\mathrm{CO}$ diffusion on $\mathrm{Pt}(111)$ with time-resolved infrared-pulsed molecular beam methods: Critical tests and analysis. J. Chem. Phys. 1990, 93, 9113-9129.

(28) Blomberg, S.; Zetterberg, J.; Zhou, J.; Merte, L. R.; Gustafson, J.; Shipilin, M.; Trinchero, A.; Miccio, L. A.; Magaña, A.; Ilyn, M.; Schiller, F.; Ortega, J. E.; Bertram, F.; Grönbeck, H.; Lundgren, E. Strain Dependent Light-off Temperature in Catalysis Revealed by Planar Laser-Induced Fluorescence. ACS Catal. 2016, 7, 110-114. 